\title{
High Resolution 3D and 4D Characterization of Microstructure Formation in Novel Ti Alloys for Additive Manufacturing
}

\author{
G. Requena ${ }^{1,2}$, Pere Barriobero-Vila ${ }^{1}$, Katrin Bugelnig ${ }^{1}$, Jan Haubrich ${ }^{1}$, Joachim Gussone ${ }^{1}$, Ulrike \\ Hecht $^{3}$, Federico Sket ${ }^{4}$, Julio Cesar da Silva ${ }^{4}$, Julie Villanova ${ }^{4}$ \\ 1. German Aerospace Center, Institute of Materials Research, Linder Höhe 51147, Cologne, Germany \\ 2. Metallic Structures and Materials Systems for Aerospace Engineering, RWTH Aachen University, \\ Aachen 52062, Germany \\ 3. Access e.V., Intzestr. 5, 52072 Aachen, Germany \\ ${ }^{4}$ IMDEA Materials Institute, C/Eric Kandel 2, 28906 Getafe, Spain; federico.sket@imdea.org \\ ${ }^{5}$ European Synchrotron Radiation Facility (ESRF), Avenue des Martyrs 71, Grenoble 38000, France \\ * Corresponding author: Guillermo.Requena@dlr.de
}

Additive manufacturing (AM) is leading a paradigm change across multiple industries such as the aerospace, biomedical and automotive sectors. Nevertheless, the vast majority of the alloys used nowadays for AM are based on compositions inherited from conventional manufacturing. As are result, complex and costly process chains are usually necessary to adapt the materials to the foreseen application. It is therefore essential to create new alloys to exploit the metallurgical conditions of AM and achieve full maturity of this technology. In this context, we are currently endeavored in the development of several Ti alloys for laser-based AM, starting from the alloy design up to component testing. High resolution 3D and 4D synchrotron characterization methods are playing a decisive role to unravel the mechanisms governing the microstructure formation of these novel materials.

A critical issue for acceptance and certification of AM components is their mechanical isotropy. Typically, epitaxial grain growth occurs across solidified layers during AM owing to the inherent directional and steep thermal gradient in the molten metal pool [1]. As a consequence, in Ti alloys, the as-built AM microstructures are characterized by coarse columnar prior $\beta$ grains with strong $<100>\beta$ orientation along the building direction, normal to the synthesized powder layers. Recently, we discovered a phase transformation path in the binary system Ti-La that opens up an alternative to avoid epitaxial-growth-driven crystallographic texture [2]. This path allows obtaining equiaxed microstructures by heterogeneous nucleation of $\alpha$ via a peritectic $\mathrm{L} 1+\beta \rightarrow \alpha$ reaction. We investigated the microstructure formation in situ by means of synchrotron tomography at the temperatures at which this reaction takes place. The experiments were carried out at the beamline ID16B of the European Synchrotron Radiation Facility (ESRF) using a voxel size of $(60 \mathrm{~nm})^{3}$ and a dedicated furnace. Fig. 1 shows exemplarily the results obtained during isothermal heat treatment at $900{ }^{\circ} \mathrm{C}$ of a Ti-Fe-La alloy produced by laser-based additive manufacturing. The reconfiguration of the microstructure as a function of time can be clearly observed, resulting in equiaxed $\alpha$ grains (dark grey) with the $\beta$ phase forming at the $\alpha$ grain boundaries.

In a further project we are dealing with the development of high strength alloys with ultrafine eutectic microstructures. The microstructure of eutectic alloys displays features of distinct length scale, i.e. dendrites of size "D" embedded in a eutectic with inter-phase spacing " $\lambda$ ". Both $D$ and $\lambda$ depend on the growth conditions during solidification and commonly decrease with increasing growth velocity or cooling rate. This gives rise to significant size strengthening effects and opens a gateway to materials design by rapid solidification. AM and, particularly, selective laser melting (SLM) are rapid solidification technologies "par excellence" which offer fast solidification/cooling rates which are nearly constant throughout an entire component owing to the layer-wise building strategy. We are developing a 
Ti-32.5wt\%Fe-based eutectic alloy by SLM aiming at obtaining ultrafine eutectic microstructures that can provide high strength and ductility. The characterization of eutectic microstructures requires 3D methods owing to the complex morphologies and connectivities exhibited by the constituent phases. Moreover, 3D characterization of ultrafine eutectics requires the use of high spatial resolutions, well below $\mu \mathrm{m}$-level. We have therefore applied near- and far-field synchrotron ptychographic tomography to study a Ti-32.5wt\%Fe alloy produced by SLM. The experiments have been carried out at the beamline ID16A of the ESRF using a voxel size of $(10 \mathrm{~nm})^{3}$. Fig. 2 shows the 3D microstructure of the Ti-32.5wt\%Fe alloy. The left figure shows the reconstructed ptychographic tomography volume with the TiFe (light grey) and the Ti (dark grey) phases clearly revealed, while the colour-coded volume on the right shows the 3D thickness of the TiFe network.
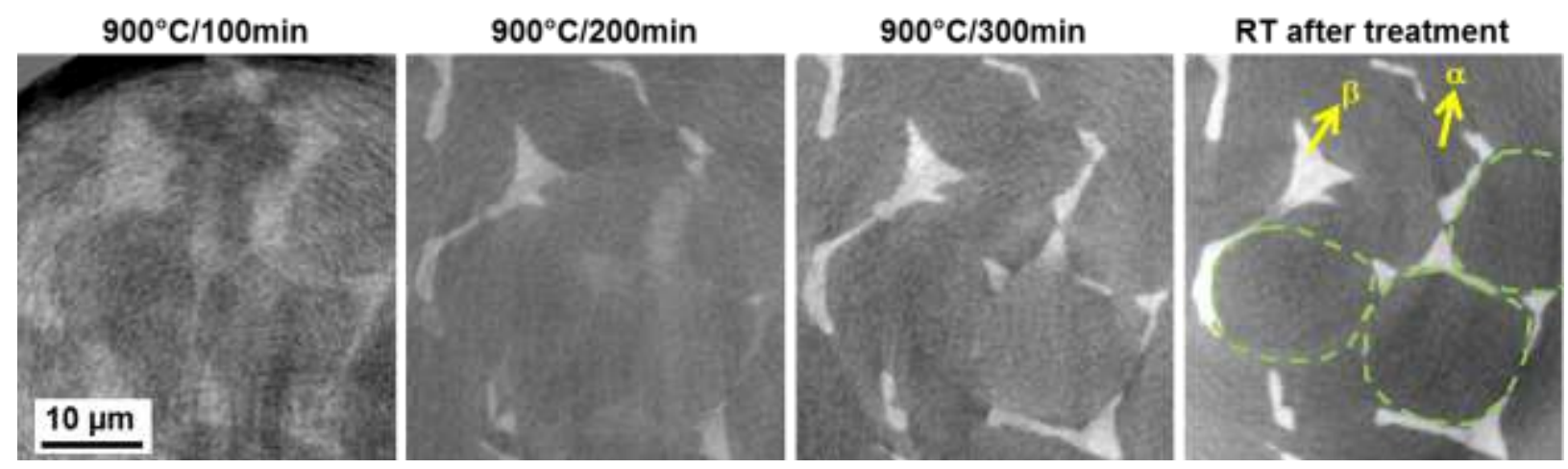

Figure 1. In situ synchrotron tomography during isothermal heat treatment at $900{ }^{\circ} \mathrm{C}$ of a Ti-Fe-La alloy produced by SLM. Beamline ESRF/ID16B, voxel size $=\left(60 \mathrm{~nm}^{3}\right)$.
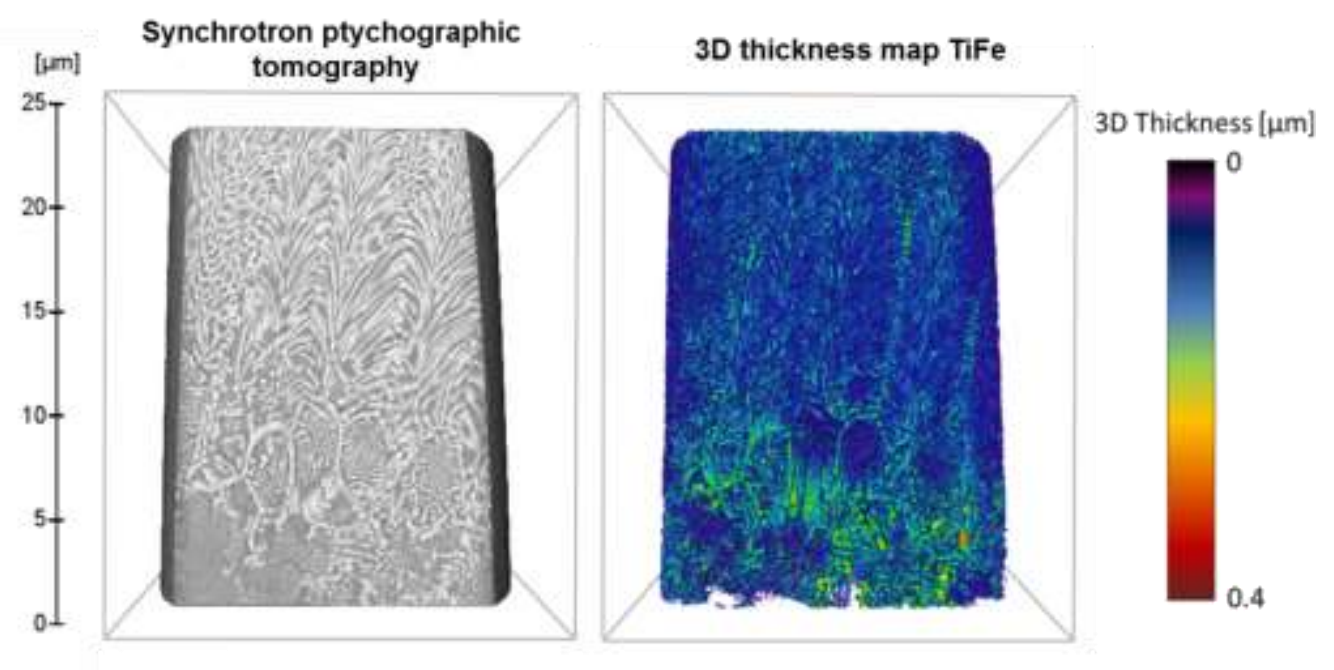

Figure 2. Eutectic Ti-32.5wt\%Fe alloy produced by SLM. Left: near-field ptychographic tomography revealing the TiFe (light grey) and the Ti (dark grey) phases. Right: 3D thickness of the TiFe network.

\section{References:}

[1] B. Dutta, F.H. Froes, Additive Manufacturing (AM) of Titanium Alloys (Elsevier, Oxford, 2015)

[2] P. Barriobero-Vila, J. Gussone, A. Stark, N. Schell, J. Haubrich and G. Requena, Nature

Communications 9 (2018) 3426P. 